\title{
The Amnioscope Strikes Back as a Useful Device for Pinhole Amniotomy in the Management of Polyhydramnios
}

\author{
Shinsuke Koyama, Ph.D., M.D., 1,2 Takuji Tomimatsu, M.D., 1,2 \\ Takeshi Kanagawa, M.D., 1,2 Tateki Tsutsui, M.D.,,2 and Tadashi Kimura, M.D. ${ }^{1,2}$
}

Polyhydramnios is associated with many serious maternal complications such as placental abruption or cord prolapse at rupture of membranes, uterine dysfunction at delivery, and postpartum hemorrhage. When considering uterine dysfunction caused by overstretched uterine muscles, active artificial amniotomy for more efficient labor seems to be a preferred obstetric management, but the potential adverse complications make obstetricians hesitate to perform this procedure. In such a challenging situation, a new strategy is required. We recently performed pinhole artificial amniotomy using an amnioscope in four women with polyhydramnios, not only to accelerate of labor but also to more slowly and safely reduce amniotic fluid volume. We had no complications using this procedure, and all women were able to have a vaginal delivery without postpartum hemorrhage and neonatal asphyxia. Pinhole artificial amniotomy using an amnioscope may be more convenient and safer than conventional artificial amniotomy. The significance of the amnioscope has been practically nil in modern obstetric management. In this pilot clinical study, we identified a new value for the amnioscope as a promising device for safer amniotomy in women with polyhydramnios.

KEYWORDS: Amnioreduction, amnioscope, amniotomy, polyhydramnios

Polyhydramnios is a condition of excessive amniotic fluid, defined as the amniotic fluid index (AFI) of greater than $25 \mathrm{~cm}$ or the single deepest pocket of larger than $8 \mathrm{~cm}$, and is identified in $\sim 1 \%$ of all pregnancies. ${ }^{1}$ This condition is divided into the idiopathic and pathological conditions. Pathological polyhydramnios is associated with fetal congenital malformations of the gastrointestinal tract or central nervous system, fetal hyperdynamic state (fetal anemia, fetal or placental hypervascular tumors, twin-to-twin transfusion syn- drome, etc.), or maternal diabetes. ${ }^{2}$ Serious maternal complications associated with polyhydramnios are placental abruption, uterine dysfunction, and postpartum hemorrhage. ${ }^{3}$ Following a rupture of membranes and an acute outflow of copious amniotic fluid, the placenta may separate from the uterine wall due to a rapid decompression of the uterine cavity before delivery of the fetus. ${ }^{1}$ Moreover, overstretched uterine muscles may cause weak labor and uterine atony, which lead to a fourfold increase in cesarean deliveries due to dystocia
${ }^{1}$ Division of Obstetrics and Gynecology, ${ }^{2}$ Department of Specific Organ Regulation, Osaka University Graduate School of Medicine, Osaka, Japan.

Address for correspondence and reprint requests: Shinsuke Koyama, Ph.D., M.D., Assistant Professor, Division of Obstetrics and Gynecology, Department of Specific Organ Regulation, Osaka University Graduate School of Medicine, 2-2 Yamadaoka, Suita, Osaka 565-0871, Japan (e-mail: skoyama@gyne.med.osaka-u.ac.jp).
Am J Perinatol Rep 2011;1:99-104. Copyright (C) 2011 by Thieme Medical Publishers, Inc., 333 Seventh Avenue, New York, NY 10001, USA. Tel: +1 (212) 584-4662.

Received: April 20, 2011. Accepted after revision: June 20, 2011. Published online: August 2, 2011.

DOI: http://dx.doi.org/10.1055/s-0031-1285983.

ISSN 2157-6998. 
and a sixfold increase in postpartum hemorrhage, respectively. ${ }^{4}$ When considering an insufficient uterine contraction and poor labor progress, active artificial amniotomy for more effective labor appears to be the preferred obstetric approach in women with polyhydramnios. However, the potential risk for serious complications such as placental abruption and cord prolapse makes obstetricians hesitate to perform artificial amniotomy. In such a challenging situation, we recently performed pinhole artificial amniotomy using an amnioscope in our institute. This procedure may be more convenient and lower risk than conventional artificial amniotomy. Historically speaking, amnioscopy had played a role in obstetric examinations to screen for meconium-stained amniotic fluid or to evaluate fetal conditions via fetal scalp blood sampling. ${ }^{5,6}$ Nowadays, the diagnostic utility of the amnioscope in various clinical conditions has been rejected time and again, losing its significance as an obstetric instrument. To our knowledge, there have been no reports describing artificial amniotomy using amnioscopes to reduce worrisome complications in polyhydramnios. In this article, we present the results of our pilot clinical study and reevaluate the amnioscope for a return to obstetric practice.

\section{MATERIALS AND METHODS}

Previously, we performed artificial amniotomy in women with polyhydramnios using a vaginal speculum. However, in the prolonged latent phase of labor frequently seen in polyhydramnios, we cannot clearly identify the forebag of the amniotic sac through a vaginal speculum because of disturbances of the thick circumferential lip of the cervix and vaginal wall in most



Figure 1 Vaginal speculum examination at 3-cm cervical dilatation. Visual field of birth canal was quite poor due to inadequate dilatation of the uterine cervix and bilateral vaginal wall compression. The forebag of the amniotic sac was completely invisible.

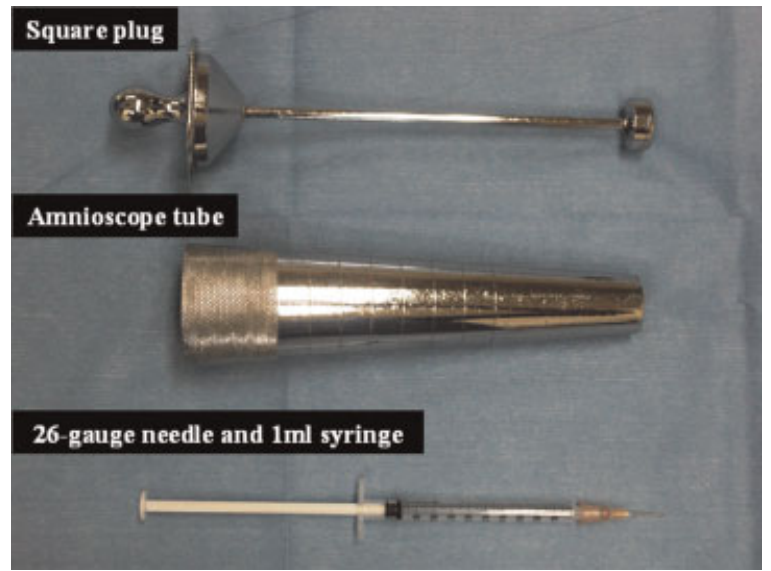

Figure 2 Instruments used for pinhole artificial amniotomy. Amnioscope tube is inserted into the vagina with a square plug inside to not injure the vaginal wall during the procedure. A 26-gauge needle is used for pinhole amniotomy.

cases (Fig. 1). Therefore, we came up with the idea that pinhole artificial amniotomy using an amnioscope with a 26-gauge needle makes it possible for us to perform amniotomy more easily and safely (Fig. 2). From January to March in 2011, we performed pinhole artificial amniotomy using an amnioscope in four women with polyhydramnios at Osaka University Hospital. We received written informed consent from each patient before the procedure. The amnioscopes used in this study have been left unused in the obstetric ward for such a long time that the manufacturer of the amnioscope is completely unknown. In this study, the causes of polyhydramnios were all associated with fetal pathological conditions as follows: tetrasomy $12 p$, diaphragmatic hernia, large cleft lip and cleft palate, and jejunal atresia.

Next, we concretely explained the procedure. We laid the patient on a delivery bed in a lithotomy position. Before and during the procedure, we continuously confirmed a reassuring fetal status by fetal heart rate monitoring and a fetal vertex position without a funic presentation by transabdominal ultrasonography. After an adequate sterilization of patient's vulva and vaginal wall, we manually lifted the anterior lip of the cervix up with the index and middle fingers during a pelvic examination, inserted an amnioscope into the vagina through the space between the fingers, and attached the tip of an amnioscope to the forebag of the amniotic sac. This allowed the forebag of the amniotic sac to become visible through the amnioscope without any disturbances (Fig. 3). We carefully performed pinhole artificial amniotomy using a 26-gauge needle with an extended 1-mL syringe (Fig. 4), and amniotic fluid started trickling through the pinhole in the amniotic membranes (Fig. 5). During pinhole amniotomy, we punctured the forebag of the amniotic 




Figure 3 A view of the forebag of the amniotic sac through an amnioscope. We inserted an amnioscope into the vagina through the space between the fingers and attached the tip of the amnioscope to the forebag of the amniotic sac. The forebag of the amniotic sac was visible through an amnioscope tube.

sac once or few times depending on the flow speed of amniotic fluid, which was ideally a couple of dozen milliliters per minute. After a few hours of the procedure, we performed complete amniotomy following confirmation of adequate amnioreduction and the engagement of the fetal head into the pelvis.

In this preliminary study, we performed artificial amniotomy using this method in four women with polyhydramnios and analyzed the obstetric outcome.

\section{RESULTS}

Detailed clinical information of these four deliveries is presented in Table 1 . In all cases, pinhole artificial amniotomy using an amnioscope was successfully per-

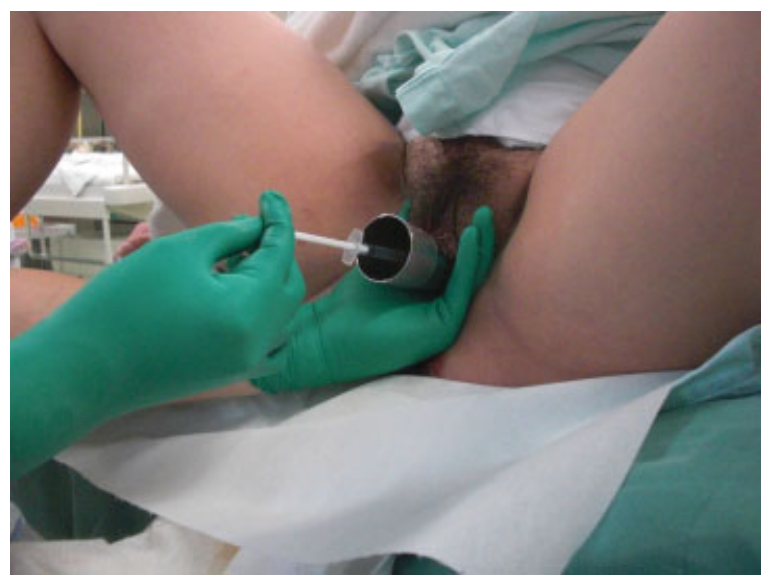

Figure 4 Actual operation of pinhole artificial amniotomy using an amnioscope. We carefully performed artificial amniotomy using a 26-gauge needle with an extended 1-mL syringe and made from one to several pinholes in the amniotic membranes.

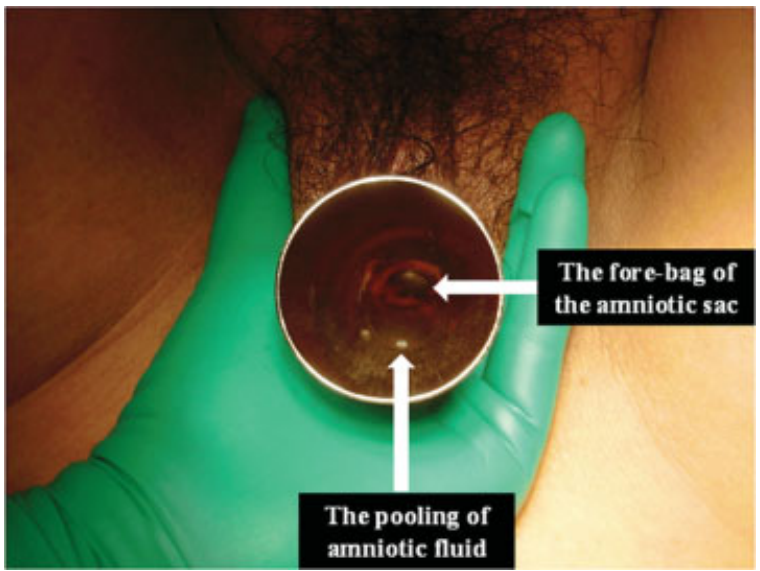

Figure 5 A view after pinhole artificial amniotomy through an amnioscope. Amniotic fluid started trickling through pinholes in the amniotic membranes, pooling in the amnioscope tube.

formed without any problems. There were no deliveries complicated by prolonged labor or postpartum hemorrhage. The results of blood gas of umbilical artery testing showed good neonatal condition at birth. To make the process of this procedure clearer, we present the detailed clinical course of case 1 in Table 1 as follows.

In case 1 , we induced labor to terminate the pregnancy at 37 weeks' gestation to relieve maternal discomfort due to polyhydramnios. After mechanical cervical ripening using a transcervical balloon catheter, intravenous oxytocin administration was started. At the beginning of induction of labor, the cervix was dilated $3 \mathrm{~cm}$, the AFI was $50 \mathrm{~cm}$, and the fetal head was floating on the cervix. During the first 4 hours of labor, cardiotocography revealed uterine dysfunction due to polyhydramnios. Therefore, we performed pinhole artificial amniotomy using an amnioscope, and slow amnioreduction was started. We punctured the forebag of the amniotic sac twice. Three hours later, we performed complete amniotomy following confirmation of adequate amnioreduction (AFI $19 \mathrm{~cm}$ ) and the engagement of the fetal head into the pelvis at $5-\mathrm{cm}$ cervical dilatation. Subsequently, the expulsive force was gradually improved, labor proceeded to active phase, and the patient delivered a $2800-\mathrm{g}$ infant vaginally in 7 hours after the initial pinhole artificial amniotomy. Total delivery time was 11.1 hours, and total blood loss at delivery was only $210 \mathrm{~mL}$.

We performed pinhole artificial amniotomy using the amnioscope in the same fashion for the three other women with polyhydramnios. As a result, we had no complications related to the use of this procedure, and all women had vaginal delivery without postpartum hemorrhage and neonatal asphyxia. 




\section{DISCUSSION}

Amniotomy has many benefits even in the normal course of labor. The presumed advantages are the acceleration of spontaneous labor, the shortening of labor without adverse perinatal outcome, the opportunity to apply a scalp electrode and an intrauterine pressure catheter for monitoring, and early detection of meconium-stained amniotic fluid. ${ }^{7}$ During amniotomy, it is important to take special care not to float the fetal head from the pelvis to avoid umbilical cord prolapse. ${ }^{8}$ Fundal pressure to fix the fetal head into the pelvis may reduce the risk of cord prolapse. $^{7}$ In polyhydramnios, the incidence of cord prolapse following amniotomy is higher because the overdistended uterus makes it difficult to palpate and fix the fetus during the procedure, and the rapid outflow of excessive amniotic fluid additionally promotes cord prolapse. Furthermore, acute uterine decompression following amniotomy may induce placental abruption. Some obstetricians recommend that slow removal of amniotic fluid by transabdominal amniocentesis helps to avoid those dangerous complications, ${ }^{1}$ but it is clinically difficult to perform amniocentesis during active labor with frequent uterine contractions. Other obstetricians advocate several membrane punctures by thin needle with direct visualization of membranes using a vaginal speculum, ${ }^{7}$ but it is technically hard to puncture membranes if there is inadequate dilatation of the uterine cervix at a latent phase of labor or poor visualization of the birth canal by bilateral vaginal wall compression, especially in the case of maternal obesity. In contrast, pinhole artificial amniotomy using an amnioscope makes it possible to reduce the amniotic fluid volume at a slow space during active labor, and it is easy to visualize the forebag of the amniotic sac without obstruction, even in case of a latent phase of labor or maternal obesity. In short, this procedure is an optimal and convenient method for artificial amniotomy in the case of polyhydramnios.

Approximately 40 years ago, the amnioscope was in the limelight as an obstetric device to detect meconium-stained amniotic fluid. ${ }^{9}$ At that time, obstetricians realized that the presence of meconium-stained amniotic fluid is an anxiety-causing sign for predicting fetal distress or asphyxia. The relationship between meconium-stained amniotic fluid and fetal asphyxia was a much-debated subject to reduce perinatal mortality and morbidity, especially in postdate pregnancy. ${ }^{10-12} \mathrm{Am}-$ nioscopes have actively been investigated in clinical research, but studies have been inconclusive. Recent studies show the mechanism of fetal passage of meconium is mainly divided into three categories: physiologically normal gastrointestinal tract maturation, increased bowel peristalsis caused by vagal reflex from common umbilical cord compression, and a pathological condition reflecting fetal compromise. ${ }^{13}$ The overall incidence of meconium-stained amniotic fluid is $12 \%$, and the rates of meconium-stained amniotic fluid increase from 1.2\% 
at 32 weeks to almost $100 \%$ at 42 weeks. ${ }^{14}$ Nowadays, it is well known that only a few cases of meconium-stained amniotic fluid are linked to fetal compromise. ${ }^{13}$ On the other hand, another discussion about thick meconiumstained amniotic fluid has recently been debated. ${ }^{15}$ Thus, an amnioinfusion to dilute thick meconium has been advocated as a procedure to reduce the incidence of meconium aspiration syndrome and improve neonatal outcomes. However, the American College of Obstetricians and Gynecologists concluded that routine prophylactic amnioinfusion is not recommended because Fraser's large randomized controlled study has not found benefits in neonatal outcome and several maternal deaths have been associated with amnioinfusion. ${ }^{16,17}$ From these considerations, the detection of meconium-stained amniotic fluid currently has little impact on obstetric management. Consequently, amnioscopy to screen for meconium-stained amniotic fluid has never had a place in routine clinical practice.

In the management of pregnancy complicated by idiopathic thrombocytopenia, fetal scalp blood sampling using amnioscopy to determine the fetal platelet count during labor has been reported. ${ }^{18}$ At that time, if fetal thrombocytopenia was verified, cesarean delivery was anecdotally preferred for preventing fetal intracranial hemorrhage. However, this procedure is not only technically difficult but also significantly inaccurate with frequent underestimation of the fetal platelet count due to in vitro coagulation during the procedure. ${ }^{19}$ Furthermore, it is hard to imagine that cesarean delivery is beneficial for the fetus because labor is already in an active phase at the time of the procedure. So, current accepted management is to select the mode of delivery only by obstetric indications without any procedure for determining fetal platelet count in utero. ${ }^{20}$ The value of this procedure renders its use moot.

Once, fetal scalp blood $\mathrm{pH}$ sampling played a valuable role in the interpretation of electronic fetal heart rate monitoring for assessment of fetal well-being during labor. ${ }^{6}$ Then, the complementary usage of this procedure with cardiotocography was expected to reduce the rate of cesarean delivery in the setting of nonreassuring fetal heart rate pattern. ${ }^{7}$ However, the cervix needs to be dilated at least 4 to $5 \mathrm{~cm}$ and vertex at a-1 station or below for success. ${ }^{8}$ Furthermore, this procedure is cumbersome, is fraught with technical error, and needs repeated testing during labor. Goodwin et al indicated that fetal scalp blood $\mathrm{pH}$ sampling has been virtually eliminated without an increase both of cesarean delivery rate due to fetal distress and of perinatal asphyxia. ${ }^{21}$ This procedure is now rarely used in standard obstetric care.

In conclusion, the significance of amnioscopy to detect meconium-stained amniotic fluid or to assist fetal scalp blood sampling has been practically nil in modern obstetric management. In this article, we describe a new use for the amnioscope as a promising device for low-risk amniotomy in women with polyhydramnios. Of course, further clinical investigation is needed to prove the utility of the amnioscope for this purpose and to search for more effective and safer management of polyhydramnios.

\section{REFERENCES}

1. Cunningham FG, Leveno KJ, Bloom SL, Hauth JC, Rouse DJ, Spong CY. Disorders of Amnionic Fluid Volume. In: Cunningham FG, Leveno KJ, Bloom SL, Hauth JC, Rouse DJ, Spong CY, eds. Williams Obstetrics, 23rd ed. New York: McGraw-Hill; 2010:490-499

2. Magann EF, Chauhan SP, Doherty DA, Lutgendorf MA, Magann MI, Morrison JC. A review of idiopathic hydramnios and pregnancy outcomes. Obstet Gynecol Surv 2007;62: 795-802

3. Nobile de Santis MS, Radaelli T, Taricco E, Bertini S, Cetin I. Excess of amniotic fluid: pathophysiology, correlated diseases and clinical management. Acta Biomed 2004;75(Suppl 1): 53-55

4. Harman CR. Amniotic fluid abnormalities. Semin Perinatol 2008;32:288-294

5. Raboni S, Kaibura CT, Fieni S. Amnioscopy: is it actual? Acta Biomed 2004;75(Suppl 1):59-61

6. Perkins RP. Requiem for a heavyweight: the demise of scalp blood pH sampling. J Matern Fetal Med 1997;6:298-300

7. Cunningham FG, Leveno KJ, Bloom SL, Hauth JC, Rouse DJ, Spong CY. Labor induction. In: Cunningham FG, Leveno KJ, Bloom SL, Hauth JC, Rouse DJ, Spong CY, eds. Williams Obstetrics, 23rd ed. New York: McGraw-Hill; 2010:500-510

8. Garite TJ. Intrapartum fetal evaluation. In: Gabbe SG, Niebyl JR, Simpson JL, eds. Obstetrics: Normal and Problem Pregnancies, 5th ed. Philadelphia: Churchill Livingstone; 2007:364-395

9. Hashimoto T. Early diagnosis of meconium stain of amniotic fluid during labor with amnioscope. J Jpn Obstet Gynecol Soc 1967;14:236-240

10. Amnioscopy M-HE. Clin Perinatol 1974;1:81-86

11. Munday P, Hamlett JD. Recognition of meconium staining of the liquor aminii at amnioscopy. Am J Obstet Gynecol 1975;122:732-733

12. Saldana LR, Schulman H, Lin C. Routine amnioscopy at term. Obstet Gynecol 1976;47:521-524

13. Ahanya SN, Lakshmanan J, Morgan BL, Ross MG. Meconium passage in utero: mechanisms, consequences, and management. Obstet Gynecol Surv 2005;60:45-56; quiz 73-74

14. Oyelese Y, Culin A, Ananth CV, Kaminsky LM, Vintzileos A, Smulian JC. Meconium-stained amniotic fluid across gestation and neonatal acid-base status. Obstet Gynecol 2006;108:345-349

15. $\mathrm{Xu} \mathrm{H,} \mathrm{Hofmeyr} \mathrm{J,} \mathrm{Roy} \mathrm{C,} \mathrm{Fraser} \mathrm{WD.} \mathrm{Intrapartum}$ amnioinfusion for meconium-stained amniotic fluid: a systematic review of randomised controlled trials. BJOG 2007;114:383-390

16. ACOG Committee Obstetric Practice. ACOG Committee Opinion Number 346, October 2006: amnioninfusion does not prevent meconium aspiration syndrome. Obstet Gynecol 2006;108:1053 
17. Fraser WD, Hofmeyr J, Lede R, et al; Amnioinfusion Trial Group. Amnioinfusion for the prevention of the meconium aspiration syndrome. N Engl J Med 2005;353:909-917

18. Scott JR, Cruikshank DP, Kochenour NK, Pitkin RM, Warenski JC. Fetal platelet counts in the obstetric management of immunologic thrombocytopenic purpura. Am J Obstet Gynecol 1980;136:495-499

19. Adams DM, Bussel JB, Druzin ML. Accurate intrapartum estimation of fetal platelet count by fetal scalp samples smear. Am J Perinatol 1994;11:42-45
20. Sukenik-Halevy R, Ellis MH, Fejgin MD. Management of immune thrombocytopenic purpura in pregnancy. Obstet Gynecol Surv 2008;63:182-188

21. Goodwin TM, Milner-Masterson L, Paul RH. Elimination of fetal scalp blood sampling on a large clinical service. Obstet Gynecol 1994;83:971-974 